\title{
Avaliação de Empatia em Residentes de Especialidades Clínicas e Cirúrgicas da Universidade Federal de São Paulo
}

\author{
Assessment of Empathy in Medical Residents \\ of Clinic and Surgical Specialties at the \\ Federal University of São Paulo
}

Caio Vinícius Suartz

Maria Inês Quintana

Ana Cecília Lucchese

Mario Alfredo De Marco ${ }^{I}$

PALAVRAS-CHAVE:

- Empatia;

- Residência;

- Especialidades.

- Psicologia;

- Relação Médico-Paciente;

- Educação Médica.

KEYWORDS:

- Empathy;

- Residence

- Specialties;

- Psychology;

- Doctor Patient Relationship;

- Medical Education.

Recebido em: 11/04/2012

Reencaminhado em: 27/02/2013

Aprovado em: 20/05/2013

REVISTA BRASILEIRA DE EDUCAÇ̄̃o MÉDICA

\section{RESUMO}

No campo da saúde, a empatia é definida como um atributo com dimensões emocionais e cognitivas que possibilita uma compreensão das experiências interiores e da perspectiva do paciente como um indivíduo singular, somada à capacidade de comunicar esse entendimento ao paciente. A função da empatia médica seria identificar e compreender os sentimentos do doente, promovendo aumento na confiança, na lealdade e no respeito entre médico e paciente. Este estudo teve como meta avaliar a empatia de residentes entre o terceiro e o quinto ano da Universidade Federal de São Paulo, buscando verificar as possíveis diferenças entre a empatia dos clínicos e cirurgiões e sua relação com o perfil profissional.

\section{ABSTRACT}

In the field of health, empathy is defined as an attribute with cognitive and emotional dimensions that enables an understanding of inner experience and a perspective of the patient as a unique individual, plus the ability to communicate this understanding to the patient. The role of empathy is to identify and understand the patient's feelings, thus promoting an increase in trust, loyalty and respect between doctor and patient. Therefore, the following study was aimed at evaluating the empathy 3rd to 5 th year medical residents from the Federal University of São Paulo to verify any possible differences between the empathy of physicians and surgeons and their relationship with the professional profile. 


\section{INTRODUÇÃO}

A palavra "empatia" deriva do grego empatheia (em = dentro e pathos $=$ sofrimento ou sentimento) e designava a relação emocional entre o autor-cantor e seu público. Em sua conceituação moderna, é uma tradução do termo alemão einfühlung, introduzido no vocabulário da experiência estética para designar a relação entre a obra de arte e seu observador, que imaginativamente se projeta no objeto contemplado. O termo ganhou um significado dissociado da arte e mais próximo do âmbito da relação pessoal ao ser traduzido para o inglês por Titchener, com o nome de empathy, cujo significado era de que, por meio da imitação interior ou esforço mental, seria possível conhecer a consciência de outra pessoa ${ }^{1,2,3}$. Recentemente, os pesquisadores têm considerado a empatia como uma capacidade multidimensional que abrange componentes cognitivos, afetivos e comportamentais ${ }^{4,5}$.

Segundo Carr et al. ${ }^{6}$, "a empatia cumpre um papel social fundamental, tornando possível o compartilhamento de experiências, necessidades e objetivo entre os indivíduos". Isto porque a habilidade de entender as intenções e as crenças do outro é crucial para o sucesso das interações sociais ${ }^{7}$.

Essa habilidade é tão importante que foi constatado que homens e mulheres que conseguem estabelecer uma relação empática com seus pares obtêm maior satisfação conjugal, na medida em que há maior compreensão na interação, diminuindo as chances de separação, aumentando o afeto e aliviando os medos e receios da outra pessoa ${ }^{8,9,10}$.

Na esfera das relações no campo da saúde, a empatia é definida como um atributo com dimensões emocionais e cognitivas que possibilita uma compreensão das experiências interiores e da perspectiva do paciente como um indivíduo singular, somada à capacidade de comunicar esse entendimento ao paciente ${ }^{11}$.

A profundidade da relação médico-paciente está vinculada a quatro elementos principais: conhecimento, confiança, lealdade e respeito ${ }^{12}$. No entanto, o dinamismo dos serviços de saúde e os avanços dos meios complementares de diagnóstico tendem a distanciar o médico da interação com o paciente, sem enxergá-lo como pessoa ${ }^{13}$.

A função da empatia médica seria identificar e compreender os sentimentos do doente e tomar da sua perspectiva ${ }^{11}$, promovendo, assim, um aumento na confiança, na lealdade e no respeito entre médico e paciente.

Já foi descrito ${ }^{14,15,16}$ que um aumento na empatia leva a um incremento na satisfação dos pacientes com o atendimento médico, além de maior adesão ao tratamento proposto. Recentemente, um estudo ${ }^{17}$ realizado na universidade de I-Shou
(Taiwan) relacionou a empatia dos cirurgiões com a satisfação dos pacientes no pós-operatório. De acordo com o estudo, existe um efeito positivo e indireto da empatia sobre a satisfação do paciente.

O exercício da empatia representa um ganho na relação tanto para quem a exerce como para quem sofre sua influência. Um estudo ${ }^{18}$ aponta que os médicos são "curadores" mais eficazes quando conseguem estabelecer uma relação empática com seus pacientes, além de ficarem mais satisfeitos com sua prática profissional. Outro estudo aponta que os médicos que aprendem a ter relações empáticas com seus pacientes reduzem sua possível frustração com estes, o que melhora a relação médico-paciente e aumenta seu impacto terapêutico ${ }^{19}$.

Por essas razões, a empatia está vinculada fortemente a uma melhora no exercício da profissão médica, devendo ser trabalhada conscientemente pelos médicos no intuito de criar relações mais empáticas com seus pacientes.

São vários os recursos que têm sido utilizados para melhorar a empatia dos alunos e residentes de Medicina, entre os quais o uso de literatura, teatro, cinema e outras artes. A utilização destes meios permite que eles aprendam como os sentimentos são expressos e ganhem novas concepções sobre suas próprias respostas emocionais à doença e ao sofrimento ${ }^{20}$.

Estudos ${ }^{21,22,23}$ têm procurado verificar a distribuição dessa capacidade na população. Decety ${ }^{23}$, da Universidade de Chicago, submeteu profissionais ligados a áreas da saúde e um grupo controle a experiências visuais de pessoas que sentiam dor e constatou por neuroimagem que profissionais da saúde têm sua empatia à dor diminuída. Han ${ }^{21}$ verificou que as mulheres apresentam um grau maior de empatia que os homens. Palwelczyk $^{22}$ relacionou as diferenças da escolha da especialidade médica e a personalidade com o sexo, obtendo que as mulheres apresentam um grau de empatia maior do que o dos homens e que estes optam em maior número pelas especialidades cirúrgicas, enquanto as mulheres tendem para áreas clínicas.

No Brasil, uma pesquisa sobre as diferenças entre o clínico e o cirurgião ${ }^{24}$ encontrou traços de personalidade bem definidos para cada uma dessas duas grandes áreas. Enquanto o clínico teria um perfil meticuloso e calculista, o cirurgião teria seu perfil baseado em atitudes práticas e objetivas.

Nessa perspectiva de que as pessoas têm graus de empatia diferentes entre si e de que ela é necessária e proporcional à qualidade da relação médico-paciente, o objetivo deste estudo foi avaliar a empatia de residentes entre o terceiro e o quinto ano da Universidade Federal de São Paulo, buscando verificar as possíveis diferenças entre a empatia dos clínicos e cirurgiões e sua relação com o perfil profissional. 


\section{MATERIAL E MÉTODOS}

Este trabalho foi aprovado pelo Conselho Nacional de Desenvolvimento e Pesquisa (CNPQ) - protocolo 116492/2010-4 —, pelo Comitê de Ética em Pesquisa da Universidade Federal de São Paulo — protocolo 1197 / 10 — e pelo Sistema Nacional de Ética em Pesquisa (Sisnep) - CAAE-0590.0.174.000-10.

Realizou-se um estudo de corte transversal com amostra de 40 residentes cirurgiões e 40 clínicos entre o terceiro e o quinto ano de residência na Unifesp no ano de 2010. As especialidades clínicas e cirúrgicas foram separadas segundo os critérios de Machado ${ }^{25}$, que são os mesmos utilizados pelo Conselho Federal de Medicina. Tais critérios separam as especialidades em quatro tipos: cognitivas, técnico-cirúrgicas e de habilidades, intermediárias e tecnológicas/burocráticas.

As especialidades cognitivas são aquelas com contato direto com o cliente e que envolvem, fundamentalmente, o uso do conhecimento cognitivo obtido na faculdade, onde o raciocínio clínico é muito valorizado. Nessa área, temos como especialidades: Alergia e Imunoterapia, Angiologia, Cardiologia, Endocrinologia, Foniatria, Geriatria, Genética Clínica, Hanseologia, Homeopatia, Infectologia, Medicina do Trabalho, Medicina Esportiva, Medicina Geral Comunitária, Medicina Interna, Neurologia, Neurologia Pediátrica, Nutrologia, Pediatria, Pneumologia, Psiquiatria, Reumatologia, Sexologia e Tisiologia.

As especialidades cirúrgicas - Cirurgia Cardiovascular, Cirurgia da Mão, Cirurgia da Cabeça e Pescoço, Cirurgia Geral, Cirurgia Pediátrica, Cirurgia Plástica, Cirurgia Torácica, Cirurgia Vascular, Mastologia e Neurocirurgia - são enquadradas na categoria técnico-cirúrgicas e de habilidades ${ }^{25}$. Essa classe também lida diretamente com o paciente, mas não de forma direta e intensa como os clínicos. O que predomina no ofício desse especialista é o conhecimento técnico, que é sempre pontual e direto.

\section{Instrumentos}

O Inventário de Empatia ${ }^{3}$ foi construído e validado no Brasil com o propósito de medir a habilidade empática de indivíduos adultos. Suas 40 questões avaliam os quatro fatores que compõem a habilidade empática e têm como valores médios populacionais os seguintes coeficientes: Tomada de Perspectiva $=40-41$; Flexibilidade Interpessoal $=31$; Altruísmo $=22$; Sensibilidade Afetiva $=35$ (percentil 50).

Tomada de Perspectiva (TP) - é ilustrado por 12 itens e se refere à capacidade de entender a perspectiva e os sentimentos da outra pessoa, mesmo em situações que envolvam conflito de interesses, as quais demandam esforço para compreender as razões do outro antes de expressar as próprias perspecti- vas. Um escore baixo neste fator denota dificuldade para compreender as perspectivas e sentimentos da outra pessoa, especialmente em situações de conflito de interesses (exemplo: questão 10 - Antes de pedir a uma pessoa para mudar um comportamento que me incomoda, procuro me colocar no lugar dela para entender o que a leva a ter tal atitude).

Flexibilidade Interpessoal (FI) - composto por dez itens, este fator expressa a capacidade para tolerar comportamentos, atitudes e pensamentos dos outros quando são muito diferentes ou provocadores de frustração. Um escore baixo neste fator indica dificuldade em aceitar pontos de vista diferentes e tendência a se aborrecer facilmente em situações de conflito de interesses ou de frustração interpessoal (exemplo: questão 5 - Quando alguém está me confidenciando um problema, exponho minha opinião objetivamente, apontando os seus erros e acertos).

Altruísmo (AL) - reflete capacidade para sacrificar os próprios interesses com a finalidade de beneficiar ou ajudar alguém. Um escore baixo neste fator revela tendência egoísta (exemplo: questão 2 - Eu adiaria a decisão de terminar um relacionamento se percebesse que o meu par está com problemas).

Sensibilidade Afetiva (SA) - reflete sentimentos de compaixão e de interesse pelo estado emocional do outro. Um escore baixo reflete pouca atenção ou cuidado em relação às necessidades dos outros (exemplo: questão 39 - Deixo de revelar uma experiência de sucesso se percebo que a outra pessoa está triste ou com problemas).

As respostas de cada item foram numeradas de 1 a 5 , em uma escala do tipo Likert, de acordo com as frequências dos comportamentos descritos, variando desde nunca (1) até sempre (5). Há itens nos quais quanto maior a pontuação, maior a empatia e itens reversos.

A Escala de Satisfação com a Vida (ESV) ${ }^{26}$ compreende múltiplos itens, cobre um único fator, é breve e tem um formato de resposta simples. Consiste em cinco perguntas que podem ser respondidas numa escala de 1 a 7, da discordância total da frase apresentada até a concordância total. Uma pontuação maior indica maior satisfação com a vida, e vice-versa. A escala de satisfação com a vida tem 23 pontos como valor médio para os médicos do Brasil, o que indica que a classe médica no País está classificada como "ligeiramente satisfeita".

O questionário sociodemográfico construído para o presente estudo continha perguntas sobre idade, sexo, estado civil, ano da graduação, especialidade, se os pais são médicos e se cursou a graduação na Unifesp-EPM.

Na execução do estudo, primeiramente este projeto de pesquisa foi aprovado pelo Comitê de Ética em Pesquisa da Unifesp (CEP), processo $\left.n^{\circ} 1197 / 10\right)$. Após a aprovação do 
órgão regulador dos residentes (Coreme) e a divulgação de uma lista com nome, data de início da residência e e-mail, foi tentado, junto aos residentes, agendar por e-mail datas para a realização da pesquisa. Após algum tempo sem sucesso, devido a e-mails incorretos, escassez de tempo dos estudantes e bloqueios das caixas de e-mail por suspeita de spam, essa abordagem foi abandonada. A tentativa de contato com os residentes passou a ser realizada de forma direta nas reuniões de departamento, enfermarias, salas de conforto médico e após as aulas. Obtivemos dessa forma 40 voluntários residentes de Cirurgia e 40 voluntários residentes de Clínica Médica.

O questionário foi preenchido após a assinatura do termo de consentimento. Uma vez coletados os questionários, foi elaborado um banco de dados que passou por uma análise estatística feita pelo Statistical Package for the Social Sciences (SPSS). Tivemos uma primeira etapa exploratória e descritiva da amostra e uma segunda na qual foram aplicados os testes estatísticos apropriados a cada tipo de variável. Foram utilizados o qui-quadrado e o Teste $\mathrm{T}$.

\section{RESULTADOS}

Foram entrevistados ao todo 80 residentes, 40 da especialidade clínica e 40 da cirúrgica, do terceiro ao quinto ano de residência da Unifesp no ano de 2010. Somente na variável gênero houve diferença entre os grupos: o gênero masculino foi predominante entre os residentes cirurgiões (77,5\%), e o feminino entre os clínicos (72,5\%). A média de idade foi de 28,24 (DP = 1,536; máx. = 32; mín. = 26); 41,2\% (n=33) são do terceiro ano, $48,8 \%(\mathrm{n}=39)$ do quarto ano, e $10 \%(\mathrm{n}=8)$ do quinto ano. Os residentes que não têm pais médicos somam $65 \%(n=52)$, e os que não fizeram a graduação na Unifesp são $67,5 \%(n=54)$. Setenta e cinco por cento da amostra $(n=60)$ não apresentam parceiros fixos (solteiros, desquitados ou viúvos) (Tabela 1).

No inventário de empatia, a pontuação foi semelhante para ambos os grupos. Para os clínicos, a média encontrada para cada um dos fatores do inventário foi: $\mathrm{FI}=31,72$ (percentil 50 nos dados normativos do Inventário de Empatia), SA = 35,87 (percentil 50); TP = 41,27 (percentil 50) e AL = 32,95 (percentil 99). A média encontrada entre os cirurgiões para cada um dos fatores do inventário foi: $\mathrm{FI}=31,20$ (percentil 50), SA 35,02 (percentil 50); $\mathrm{TP}=42,22$ (percentil 60) e AL = 29,92 (percentil 90) (Tabela 2).

No questionário de satisfação com a vida, a média encontrada para os clínicos foi de 25,97 (DP = 5,69), estando, portanto, um pouco acima da amplitude que os classificaria como "ligeiramente satisfeitos" (21 a 25 pontos); e de 26,40 (DP = 5,69) para os cirurgiões, que está 1,40 ponto acima da amplitude que os classificaria como "ligeiramente satisfeitos" (Tabela 2).
TABELA 1

Resultados do questionário sociodemográfico

\begin{tabular}{lcc}
\hline & $\begin{array}{c}\text { Cirurgião } \\
(\mathbf{n}=40) \\
\%(\mathbf{n})\end{array}$ & $\begin{array}{c}\text { Clínico } \\
(\mathbf{n}=40) \\
\%(\mathbf{n})\end{array}$ \\
\hline $\begin{array}{l}\text { Sexo* } \\
\text { Masculino }\end{array}$ & \\
$\quad$ Feminino & $77,5(31)$ & $27,5(11)$ \\
Graduação & $22,5(9)$ & $72,5(29)$ \\
$\quad$ Unifesp & & \\
$\quad$ Outras universidades & $50,0(20)$ & $15,0(6)$ \\
Estado civil & $50,0(20)$ & $85,0(34)$ \\
$\quad$ Solteiro/separado/viúvo & & \\
Casado/ amasiado & $72,5(29)$ & $77,5(31)$ \\
Pais médicos & $27,5(11)$ & $22,5(9)$ \\
Sim & & \\
Não & $30,0(12)$ & $40,0(16)$ \\
\hline
\end{tabular}

Nota: ${ }^{*} \mathrm{p}<0,001$

TABELA 2

Resultados do Inventário de Empatia (IE) e Escala de Satisfação com a Vida (ESV)

\begin{tabular}{lcccc}
\hline & \multicolumn{2}{c}{$\begin{array}{c}\text { Cirurgiões } \\
(\mathbf{n}=\mathbf{4 0 )}\end{array}$} & \multicolumn{2}{c}{$\begin{array}{c}\text { Clínicos } \\
(\mathbf{n}=\mathbf{4 0 )}\end{array}$} \\
\hline & Média & DP & Média & DP \\
\hline Escala IE & - & - & - & - \\
Tomada de perspectiva & 42,22 & 7,767 & 41,27 & 5,570 \\
Flexibilidade interpessoal & 31,20 & 6,009 & 31,72 & 5,982 \\
Altruísmo & 29,92 & 5,712 & 32,95 & 5,178 \\
Sensibilidade afetiva & 35,02 & 5,131 & 35,87 & 4,285 \\
Escala de SV & 26,40 & 6,238 & 25,97 & 5,695 \\
\hline
\end{tabular}

Nota 1: DP — desvio padrão.

Nota 2: Obs.: Valores médios dos coeficientes apresentados $\mathrm{QV}=21-25 / \mathrm{TP}=40-41 / \mathrm{FI}=31 / \mathrm{AL}=22 / \mathrm{SE}=35$.

\section{DISCUSSÃO E CONCLUSÕES}

O estudo mostrou que há uma preponderância de homens nas especialidades cirúrgicas $(77,5 \%)$ e de mulheres nas especialidades clínicas $(72,5 \%)$. Na área clínica, existe um número consideravelmente maior de residentes advindos de outras escolas. Além disso, foi encontrada uma diferença estatisticamente significante entre o altruísmo dos clínicos e dos cirurgiões.

Em relação aos dados sociodemográficos obtidos, um estudo desenvolvido por Bellodi ${ }^{24}$, cujo objetivo era traçar o 
perfil de médicos cirurgiões e médicos clínicos, utilizando 60 residentes do Hospital das Clínicas de São Paulo (30 clínicos e 30 cirurgiões), encontrou dados semelhantes aos deste estudo. Aquele autor encontrou que $93 \%$ dos residentes clínicos e $97 \%$ dos residentes cirurgiões são solteiros, assim como os residentes da Unifesp, onde a maioria (77,5\% e 72,5\%) é solteira. Outro achado semelhante diz respeito à proporção entre homens e mulheres na residência de Cirurgia: enquanto o presente estudo encontrou que $77,5 \%$ dos residentes dessa especialidade são do sexo masculino, Bellodi ${ }^{24}$ constatou uma predominância desse sexo de $97 \%$.

A proporção do número de mulheres residentes de especialidades clínicas na Unifesp é maior do que a encontrada por Bellodi ${ }^{24}$ : 72,5\% na Unifesp e 50\% no estudo da pesquisadora. Outra diferença está fundamentada no fato de a autora ter encontrado que ambos os grupos têm, em sua maioria, pais médicos (70\% clínicos e $63 \%$ dos cirurgiões), enquanto o presente estudo revelou que a maioria dos residentes das especialidades clínicas e cirúrgicas não tem pais médicos (60\% dos clínicos e 70\% dos cirurgiões).

Quanto aos resultados do questionário de empatia, os dados dos residentes em especialidades cirúrgicas e clínicas apontam que esses médicos apresentam altruísmo acima da média da população (percentis 99 e 90, respectivamente, se comparados aos dados normativos), enquanto a sua flexibilidade interpessoal, sensibilidade afetiva e tomada de perspectiva apresentam escores compatíveis com as médias dos dados normativos do I.E.

Há uma diferença significante entre clínicos $(\mathrm{AL}=32,95$; percentil 99) e cirurgiões (AL $=29,92$; percentil 90) (teste $\mathrm{T} ; \mathrm{t}=$ $-2,481 ; \mathrm{p}=0,015)$ no quesito altruísmo. Trata-se de um componente afetivo da empatia definido como vontade de ajudar outra pessoa sem expectativa de qualquer compensação. Sua diferença entre os grupos pode indicar que há um perfil na escolha da especialidade que se acentua com a prática médica. A práxis dos clínicos, estimulando mais habilidades sociais, e a dos cirurgiões, mais aspectos técnicos, pode estar ligada a esse interesse elevado dos clínicos em auxiliar sem expectativa de compensação ${ }^{24}$.

\section{REFERÊNCIAS}

1. Burns D, Auerbach A. Therapeutic empathy in cognitive-behavioral therapy: does it really make a difference? In: Salkovskis PM, Rachman S, organizadores. Frontiers of cognitive therapy. New York: Guilford; 1996. p.135-63.

2. Wispé L. Historia del concepto de empatía. In: Eisenberg $\mathrm{N}$, Strayer J, organizadores. La empatía y su desarrollo. Bilbao: Desclée de Brouwer; 1992. p.27-48
3. Falcone E, Ferreira M, Luz R, Fernández C, Faria C, D’Augustin J, Sardinha A, Pinho V. Inventário de Empatia (IE): desenvolvimento e Validação de uma medida brasileira. Avaliação Psicológica, 7(3), pp. 321-334 ;2008.

4. Falcone EMO. A avaliação de um programa de treinamento da empatia com universitários. São Paulo; 1998. Doutorado [Tese] — Universidade de São Paulo.

5. Koller SH, Camino C, Ribeiro J. Adaptação e validação interna de duas escalas de empatia para uso no Brasil. Estud Psicol. (Campinas). 2001;18(3):45-53.

6. Carr L, Iacoboni M, Dubeau MC, Mazziotta JC, Lenzi GL. Neural mechanisms of empathy in humans: a relay from neural systems for imitation to limbic areas. Proc Natl Acad Sci USA. 2003; 100(9):5497-502.

7. Lago KC. Fadiga por compaixão: quando ajudar dói. Brasília; 2008. Mestrado [Dissertação] — Universidade de Brasília.

8. Bissonnette VL, Rusbult CE, Kilpatrick SD. Empathic accuracy and marital conflict resolution. In: Ickes W, editor. Empathic accuracy. New York: Gilford;1997. p.251-81.

9. SardinhaI A, Falcone EMO, Ferreira MC. As relações entre a satisfação conjugal e as habilidades sociais percebidas no cônjuge. Psicol Teor Pesqui. 2009;25(3):395-402. DOI: 10.1590/S0102-37722009000300013.

10. Oliveira MGS. A avaliação da empatia e da satisfação conjugal: um estudo preliminar. Rio de Janeiro; 2005. Mestrado [Dissertação] — Universidade do Estado do Rio de Janeiro.

11. Hojat M, Gonnella JS, Mangione S, Naasca TJ, Veloski JJ, Erdmann JB et al.. Empathy in medical students as related to academic performance, clinical competence and gender. Med Educ. 2002; 36(6):522-7. DOI: 10.1046/j.1365-2923.2002.01234.x.

12. Roberge D, Beaulieu M-D, Haddad S, Lebeau R, Pineault $\mathrm{R}$. Loyalty to the regular care provider: patients` and physicians`views. Fam Pract. 2001;18(1):53-9. DOI: 10.1093/ fampra/18.1.53

13. Iglehart J. Forum on the future of academic medicine: session IV-there realities of the health care environment. Acad Med. 1998;73(9):956-61.

14. Hart CN, Drotar D, Gori A, Lewin L. Enhancing parent-provider communication in ambulatory pediatric practice. Patient Educ Couns. 2006;63(1-2):38-46. DOI: 10.1016/j. pec.2005.08.007.

15. Kim SS, Kaplowitz S, Johnston MV. The effects of physician empathy on patient satisfaction and compliance. Eval Health Prof. 2004;27(3):237-51.

16. Zachariae R, Pedersen CG, Jensen AB, Ehrnrooth E, Rossen $\mathrm{PB}$, Von der Maase H. Association of perceived physician 
communication style with patient satisfaction, distress, cancer-related self-efficacy, and perceived control over the disease. Br J Cancer. 2003;88(5):658 —65. DOI: 10.1038/ sj.bjc.6600798

17. Weng HC, Steed JF, Yu SW, Liu YT, Hsu CC, Yu TJ, Chen $W$. The effect of surgeon empathy and emotional intelligence on patient satisfaction. Adv Health Sci Educ Theory Pract.2011;16(5):591-600. DOI: 10.1007/s10459-011-9278-3. 18. Larson EB, Yao X. Clinical empathy as emotional labor in the patient-physician relationship. JAMA. 2005;293(9):1100-6.

18. Halpern J. Empathy and patient-physician conflicts. J Gen Intern Med. 2007;22(5):696-700.

19. Ziółkowska-Rudowicz E, Kładna A.. [Empathy-building of physicians. Part III. Students exposure to literature, theatre, film and the arts]. Pol Merkur Lekarski. 2010;29(173):331-5. Polish.

20. Han S, Fan Y, Mao L. Gender difference in empathy for pain: an electrophysiological investigation. Brain Res. 2008;27(1196):85-93. DOI: 10.1016/j.brainres.2007.12.062.

21. Pawełczyk A, Pawełczyk T, Bielecki J. [Differences in medical specialty choice and in personality factors among female and male medical students]. Pol Merkur Lekarski. 2007 Nov;23(137):363-6. Polish.

22. Decety J, Yang CY, Cheng Y. Physicians down-regulate their pain empathy response: an event-related brain potential study. Neuroimage. 2010;50(4):1676-82. DOI: 10.1016/j. neuroimage.2010.01.025

23. Bellodi PL. O clínico e o cirurgião: estereótipo, personalidade e escolha de especialidade médica. $2^{\underline{a}}$ ed. São Paulo: Casa do Psicólogo;2001.
24. Machado MH. Os médicos no Brasil: um retrato da realidade. Rio de Janeiro: FIOCRUZ, 1997. DOI: 10.7476/9788575412695

25. Gouveia VV. Barbosa GA, Andrade EO, Carneiro MB. Medindo a satisfação com a vida dos médicos no Brasil. J Bras Psiquiatr. 2005;54(4):298-305.

\section{CONTRIBUIÇÃO DOS AUTORES}

Caio Vinícius Suartz: Elaboração da estrutura do trabalho, aplicação dos questionários, avaliação dos resultados, redação do artigo.

Maria Inês Quintana: Elaboração e correção da parte estatística do trabalho

Ana Cecília Lucchese: Elaboração da estrutura do trabalho, orientação metodológica , correção e confecção do artigo.

Mario Alfredo De Marco: Elaboração da estrutura do trabalho, orientação principal , correção e confecção do artigo.

\section{CONFLITO DE INTERESSES}

Declarou não haver.

\section{ENDEREÇO PARA CORRESPONDÊNCIA}

Rua Votuporanga 67

Perdizes - São Paulo

CEP. 01256-000 SP

E-mail: caio.v_suartz@hotmail.com 